\title{
Embedded clustering in Cr-doped AIN: Evidence for general behavior in dilute magnetic III-nitride semiconductors
}

\author{
X. Y. Cui ${ }^{\text {a) }}$ and D. Fernandez-Hevia ${ }^{\text {b) }}$ \\ School of Physics, The University of Sydney, Sydney 2006, NSW Australia \\ B. Delley \\ Paul Scherrer Institute, WHGA/123 CH-5232 Villigen PSI, Switzerland \\ A. J. Freeman \\ Department of Physics and Astronomy, Northwestern University, Evanston, Illinois 60208-3112 \\ C. Stampfl \\ School of Physics, The University of Sydney, Sydney 2006, NSW Australia
}

(Received 16 February 2007; accepted 23 March 2007; published online 31 May 2007)

\begin{abstract}
We report a systematic density-functional theory investigation of the "structure-property relationship" of Cr:AlN by doping up to $5 \mathrm{Cr}$ atoms in large supercells, for which exhaustive structural and magnetic configurations have been calculated-including full atomic relaxation. Our results demonstrate that the $\mathrm{Cr}$ atoms tend to segregate to form $\mathrm{Cr}-\mathrm{N}-\mathrm{Cr}$ bonded clusters, which are embedded in the AlN host wurtzite structure. Significantly, while the ferromagnetic state with a spin moment close to $3 \mu_{\mathrm{B}} / \mathrm{Cr}$ is the ground state for both isolated "single" and "pair" doping configurations, for larger cluster configurations states containing antiferromagnetic or ferrimagnetic coupling with net spin in the range of $0-1.53 \mu_{\mathrm{B}} / \mathrm{Cr}$ are found to be energetically more favorable. Electrical conductivity (half-metallic or insulating) is predicted to be sensitively dependent on the dopant concentration. We propose a picture that various sized $\mathrm{Cr}-\mathrm{N}-\mathrm{Cr}$ bonded clusters coexist and the statistical distribution and associated magnetic properties will depend sensitively on the growth conditions. Such a scenario is in agreement with recent experiments and can help understand a number of hitherto puzzling experimental observations, notably the low mean saturation magnetic moment, the contracted lattice constants, and the highly insulating behavior. (c) 2007 American Institute of Physics. [DOI: 10.1063/1.2735405]
\end{abstract}

\section{INTRODUCTION}

Recently, transition metal (TM)-doped III-V type diluted magnetic semiconductors (DMSs) have attracted intense attention because of their potential as spintronics devices. ${ }^{1}$ Traditional III-V DMS, such as Mn:GaAs systems, suffer from the limitation of the Curie temperature $\left(T_{c}\right)$ to low temperatures $(\sim 170 \mathrm{~K})$, which significantly circumscribes their practical use in room temperature devices. More recent studies have focused on wide band gap $\mathrm{GaN}$ or AlN-based systems because of the light column III anion, which is expected to have a reduced spin-orbit interaction and hence a longer lifetime for its polarized carriers. In addition, based on the mean-field Zener model, the smaller lattice constants, particularly for AlN, are expected to result in a larger $s p-d$ hybridization between the valence orbitals of the host semiconductor and the $d$ shells of the magnetic ions than that in $\mathrm{Mn}: \mathrm{GaAs}$. Consequently, the strength of the carrier-mediated interaction and the $T_{c}$ is expected to be significantly higher. ${ }^{2,3}$ The subsequent discovery of room temperature (up to $900 \mathrm{~K}$ ) ferromagnetism in Cr-doped AlN single crystals has stirred further interest. ${ }^{4-17} \mathrm{Cr}$-doped AlN thin films have been grown successfully using a variety of techniques, such

\footnotetext{
${ }^{a)}$ Corresponding author; electronic mail: carlc@physics.usyd.edu.au

${ }^{b}$ Present address: Universidad Politecnica de Madrid, Spain.
}

as reactive sputtering, ${ }^{4-7}$ ion implantation, ${ }^{8,9}$ and molecular beam epitaxy. ${ }^{10-14}$ There are also several first-principles theoretical studies on Cr:AlN. ${ }^{10,15-17}$

Despite these intense efforts, however, many fundamental questions remain unanswered. For example, the observed magnetic moment, typically varying between 0.5 $-1.1 \mu_{\mathrm{B}} / \mathrm{Cr},{ }^{4-6,8,10-12}$ is much lower than the hitherto theoretical expectation of $3 \mu_{\mathrm{B}} / \mathrm{Cr}$ based on density-functional theory (DFT) "single-doping" investigations (assuming a homogeneous distribution with one $\mathrm{Cr}$ atom located on the $\mathrm{Al}$ site). ${ }^{10,15,16}$ The much smaller saturation magnetic moment was initially attributed to there being only a percentage of the $\mathrm{Cr}$ atoms that are "magnetically active" with a moment $3 \mu_{\mathrm{B}} / \mathrm{Cr} .{ }^{10}$ However, to our knowledge, there has been no solid evidence to support such a scenario. In the meantime, this large variation of magnetic moment between theory and experiment is serious, particularly from a theoretical point of view because (i) the magnetic moment is a fundamental property associated with the bandstructure and (ii) first-principles calculations such as DFT are expected to give rather reliable predictions of magnetic moments; typically the error bar is less than $10 \%$. In addition, the observed critical dependence of the magnetic properties on details of the sample growth, particularly the doping concentration, $5,7,10-12$ clearly suggests that the homogeneous singledoping description is incomplete. 
Recently, Wang et al. ${ }^{17}$ reported a "pair-doping" investigation by doping two $\mathrm{Cr}$ atoms in both bulk AlN using 72 atom supercells and in AlN (1120) $2 \times 2$ thin films using nine-layer slabs, and showed that $\mathrm{Cr}$ atoms couple ferromagnetically with a magnetic moment close to $3 \mu_{\mathrm{B}} / \mathrm{Cr}$. They also found that as the distance between the two $\mathrm{Cr}$ atoms increases, the ferromagnetic (FM) and the antiferromagnetic (AFM) or ferrimagnetic states become energetically degenerate; thus, they proposed that this degeneracy may account for the observed low magnetic moment per $\mathrm{Cr}$ atom. For simplicity, in this work, "AFM" states also include ferrimagnetism. We argue, however, that this explanation cannot be responsible for the observed "low-mean-magnetic-moment:" Since in both bulk and thin-film AlN systems, as the authors predicted, for the "near Cr-Cr pair" (around $3 \AA$ in both bulk and thin film), the coupling is always FM while for the $\mathrm{Cr}-\mathrm{Cr}$ pair with larger distances considered, the AFM coupling is always predicted; thus, one would expected for higher doping concentration (with shorter mean $\mathrm{Cr}-\mathrm{Cr}$ distance) that the mean magnetic moment should be increased. Conversely, however, various experiments have shown that the mean magnetic moment is strongly suppressed with increasing $\mathrm{Cr}$ concentration. ${ }^{5,7,11,12}$

In general, while the "pair model" is widely used to determine and predict the magnetic stability by comparing the relative total energy of the FM and AFM phases, there is no formal justification for not using larger models and investigating the possible complicated magnetic coupling therein. This is particularly true in dilute doped systems in which the interactions among the dopants are attractive. ${ }^{18}$ In fact, van Schilfgaarde and Mryasov ${ }^{19}$ first suggested that in TM-doped III-V semiconductors, the magnetic impurities may aggregate into small nanoclusters of a few magnetic atoms due to the large exchange energy of the TM dopants. Subsequently, we theoretically confirmed such a picture and found that the states containing AFM coupling are favorable for more than pair-Cr doping configurations in $\mathrm{Cr}: \mathrm{GaN},{ }^{20,21}$ demonstrating that such "poly doping" (in contrast to single- or pair doping) is crucial to give a correct description regarding the magnetic interactions in DMS.

Another interesting phenomenon is that, after doping $\mathrm{Cr}$ in AlN, one would expect larger lattice constants due to the larger atomic radius of $\mathrm{Cr}(1.40 \AA)$ than $\mathrm{Al}(1.25 \AA)$. However, by contrast, experiments showed decreased values. ${ }^{11,12}$ Moreover, for the electrical conductivity, while several theoretical studies predicted the appealing half-metallicity, ${ }^{10,16,17}$ experiments show that they are actually semi-insulating ${ }^{11}$ or insulating. ${ }^{14}$ The mechanisms responsible for these phenomena, particularly on the atomic scale, still remain unanswered. In fact, there is still no consensus regarding the spatial distribution of the dopants and whether the FM originates from a uniform alloy or from certain clusters, precipitates, or secondary phases.

Such a confusing situation clearly mandates a thorough investigation of "structure-property relationships" in this material. Hence, we determine the spatial distribution and magnetic coupling of $\mathrm{Cr}$-doped AlN by doping up to $5 \mathrm{Cr}$ atoms, incorporated on cation sites in large supercells with periodic boundary conditions. We demonstrate that the dopants tend to segregate to form $\mathrm{Cr}-\mathrm{N}-\mathrm{Cr}$ bonded clusters, and while for pair doping the coupling is $\mathrm{FM}$, for larger $\mathrm{Cr}$ cluster doping in AlN, states containing AFM coupling are favored, leading to a much lower magnetic moment and contracted lattice constants, in agreement with experimental observations. Our results also suggest that the Cr-rich clusters observed in experiments are unlikely to be $\mathrm{Cr}-\mathrm{Cr}$ bonded, but rather are $\mathrm{Cr}-\mathrm{N}-\mathrm{Cr}$ bonded. Based on the electronic bandstructure, we predict that the electrical conductivity (half-metallic or insulating) is sensitively dependent on the dopant concentration. The results of our study resemble those for $\mathrm{Cr}: \mathrm{GaN}$ and $\mathrm{Mn}: \mathrm{GaN}$, and we argue that such a scenario may also hold for other dilute magnetic semiconductor systems.

\section{CALCULATION DETAILS}

We perform density functional theory (DFT) calculations, using the generalized gradient approximation ${ }^{22}$ with the periodic $\mathrm{DMol}^{3}$ code ${ }^{23}$ and newly developed densityfunctional semilocal pseudopotentials DSPP. ${ }^{24}$ In this scheme, all electrons for $\mathrm{N}$ and $\mathrm{Al}$ are included, while for $\mathrm{Cr}$ the $3 s^{2} 3 p^{6} 3 d^{5} 4 s^{1}$ electrons are treated as valence electrons. A double set of numerical valence functions with a local basis cutoff $R_{c}$ of 11.0 a.u. is used. The calculated optimized wurtzite lattice constants are $a=3.136 \AA$ and $c=5.010 \AA$ with internal parameter $u=0.3822$, which are in good agreement with experimental and previous theoretical results. ${ }^{25}$ Various supercells, 32-atom $(2 a \times 2 a \times 2 c), \quad 72$-atom $(3 a \times 3 a \times 2 c), 96$-atom (cubic) $(2 \sqrt{3} a \times 3 a \times 2 c), 108$-atom $(3 a \times 3 a \times 3 c), \quad 128$-atom $(4 a \times 4 a \times 2 c)$, and 256-atom $(4 a \times 4 a \times 4 c)$ are employed. Reciprocal space k-point meshes of $8 \times 8 \times 6$ for the 32 -atom, $4 \times 4 \times 3$ for the 72 atom, and $2 \times 2 \times 2$ for the 96-, 108-, and 128-atom supercells and $2 \times 2 \times 1$ for 256 -atom-cell are employed. Large supercells ensure that for each configuration studied, the interaction between neighboring supercells is negligible, and so the results present truly isolated configurations. Convergence as a function of supercell size has been checked for all the favorable structures including full atomic relaxation. We find that the size of the supercell is crucial to determine the correct magnetic coupling and electronic structure.

To determine the relative stability of different configurations, the formation energy is calculated as

$$
E^{f}=E_{\mathrm{Cr}: \mathrm{AlN}}-E_{\mathrm{ref}-\mathrm{AlN}}-n \mu_{\mathrm{Cr}}+m \mu_{\mathrm{Al}}+l \mu_{\mathrm{N}},
$$

where $E_{\mathrm{Cr} \text { AlN }}$ and $E_{\text {ref-AlN }}$ are the total energies of Cr-doped AlN and the pure AlN reference structure (as calculated with the same size supercell), respectively. $\mu_{\mathrm{Al}}, \mu_{\mathrm{Cr}}$, and $\mu_{\mathrm{N}}$ are the atom chemical potentials of $\mathrm{Al}, \mathrm{Cr}$, and $\mathrm{N}$, while the integers $n, m$, and $l$ are the number of doped $\mathrm{Cr}$ atoms, and substituted $\mathrm{Al}$ and $\mathrm{N}$ atoms, respectively. The chemical potentials depend on the experimental conditions under which the material is grown. In order to determine these quantities, we invoke the relationship $\mu_{\mathrm{Al}}+\mu_{\mathrm{N}}=\mu_{\mathrm{AlN}}$, assuming both species are in thermal equilibrium with AlN. Furthermore, the chemical potentials must satisfy the boundary conditions $\mu_{\mathrm{N}}<1 / 2 \mu_{\mathrm{N}_{2}}$ and $\mu_{\mathrm{Al}}<\mu_{\mathrm{Al}(\text { bulk) }}$ (if this were not the case, then AlN would be thermodynamically unstable with respect to the formation of $\mathrm{N}_{2}$ molecules or bulk $\mathrm{Al}$ ). 

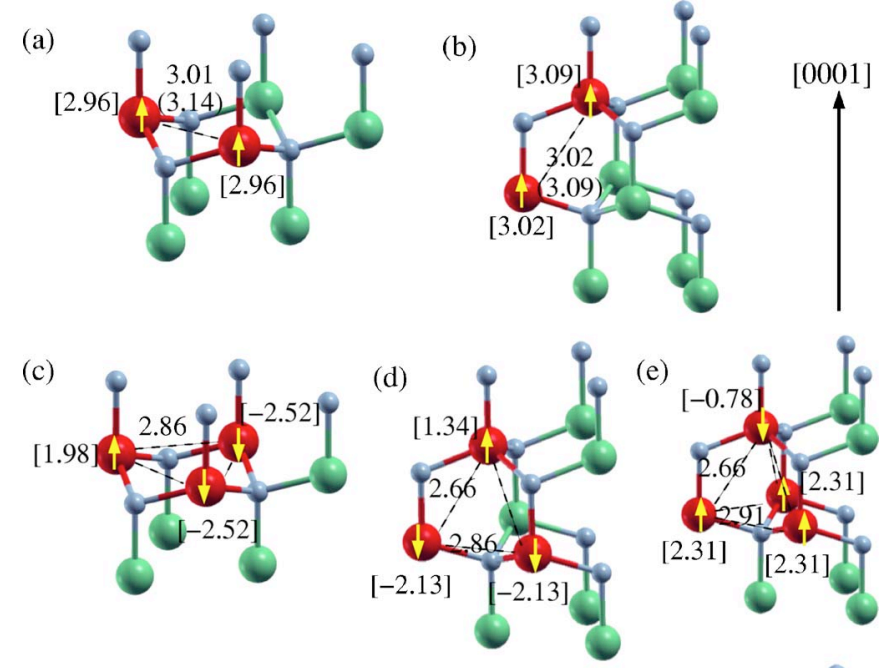

(f)

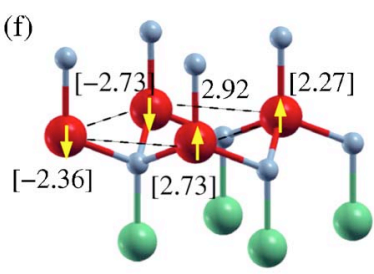

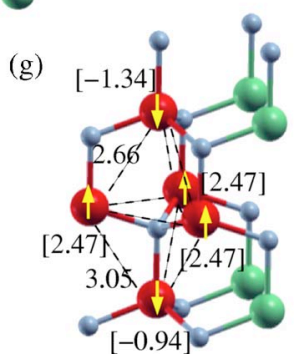

FIG. 1. (Color online) Optimized Cr clustering geometries and magnetic structures. The dark (red) circles with arrows represent substitutional $\mathrm{Cr}_{\mathrm{Al}}$, the light (green) circles $\mathrm{Al}$ atoms, and the small light gray circles $\mathrm{N}$ atoms. The $\mathrm{Cr}-\mathrm{Cr}$ distances before (in parentheses) and after relaxation are in angstrom, and the arrows indicate the directions of the local atomic spin for the magnetic ground state. The atomic spin moment values are given in square brackets in $\mu_{\mathrm{B}}$.

When imposing certain growth conditions [nitrogen-rich $\left(\mu_{\mathrm{N}}=1 / 2 \mu_{\mathrm{N}_{2}}\right)$ or aluminum-rich $\left(\mu_{\mathrm{Al}}=\mu_{\mathrm{Al}(\text { bulk })}\right)$ conditions], the chemical potential for the other species can be determined from the above (thermal equilibrium) relationship. The atomic chemical potential for $\mathrm{Cr}$ is assumed to be determined by equilibrium with bulk $\mathrm{Cr}_{2} \mathrm{~N}$, which has been detected in Cr:AlN in experiments under nonoptimized growth conditions. ${ }^{11,12}$ Under nitrogen-rich conditions, $\mu_{\mathrm{Cr}}=1 / 2\left[E_{\mathrm{Cr}_{2} \mathrm{~N}(\text { bulk })}-1 / 2 E_{\mathrm{N}_{2}}\right]$ and $\mu_{\mathrm{Al}}=E_{\mathrm{AlN}(\text { bulk })}-1 / 2 E_{\mathrm{N}_{2}}$; and under aluminum-rich conditions, $\mu_{\mathrm{N}}=E_{\mathrm{AlN}(\mathrm{bulk})}$ $-E_{\mathrm{Al}(\text { bulk })}$ and $\mu_{\mathrm{Cr}}=1 / 2\left[E_{\mathrm{Cr}_{2} \mathrm{~N}(\text { bulk })}-\left(E_{\mathrm{AlN}(\text { bulk })}-E_{\mathrm{Al}(\text { bulk })}\right)\right]$. For bulk $\mathrm{Cr}$ and bulk $\mathrm{Al}$, we used the body-centered-cubic and the face-centered-cubic structures, respectively. For bulk $\mathrm{Cr}_{2} \mathrm{~N}$, theoretical studies, our spin-polarized DFT calculations show it is nonmagnetic and the calculated heat of formation is $-0.93 \mathrm{eV}$ per formula unit with $P 63 / \mathrm{mmc}$ symmetry, ${ }^{26}$ which is very close to the experimental value of $-1.01 \mathrm{eV} .^{27}$ Further computational details can be found in Ref. 21.

\section{RESULTS}

For doping of up to $5 \mathrm{Cr}$ atoms in large supercells, we have performed an exhaustive search for the geometric and magnetic structures. Total energy calculations clearly show that the dopants prefer to occupy neighboring cation sites. For a given favorable structure containing more than one $\mathrm{Cr}$
TABLE I. Formation energies (under N-rich and Al-rich conditions), net spin values, and ground magnetic states for the favorable configurations. The quantities in bold type are for the lowest energy structures for a given number of $\mathrm{Cr}$ atoms.

\begin{tabular}{|c|c|c|c|c|c|}
\hline Structure & $\begin{array}{c}\text { No. of } \\
\text { Cr atoms }\end{array}$ & $\begin{array}{c}E_{\mathrm{N}-\text { rich }}^{f} \\
(\mathrm{eV} / \mathrm{Cr})\end{array}$ & $\begin{array}{l}E_{\text {Al-rich }}^{f} \\
(\mathrm{eV} / \mathrm{Cr})\end{array}$ & $\begin{array}{l}\text { Net spin } \\
\left(\mu_{\mathrm{B}} / \mathrm{Cr}\right)\end{array}$ & Ground state \\
\hline \multicolumn{6}{|c|}{ Single-doping configuration } \\
\hline $\mathrm{Cr}_{\mathrm{Al}}$ & 1 & 3.01 & 4.41 & 2.96 & $\cdots$ \\
\hline $\mathrm{Cr}_{\mathrm{N}}$ & 1 & 12.43 & 8.23 & 1.07 & $\cdots$ \\
\hline $\mathrm{Cr}_{i-O}$ & 1 & 9.94 & 8.54 & 0 & $\cdots$ \\
\hline $\mathrm{Cr}_{i-T}$ & 1 & 11.72 & 10.32 & 0 & $\cdots$ \\
\hline \multicolumn{6}{|c|}{$\mathrm{Cr}-\mathrm{Cr}$ bonded configurations } \\
\hline I & 2 & 7.67 & 6.27 & 0.03 & AFM \\
\hline II & 2 & 7.94 & 6.54 & 0.02 & AFM \\
\hline III & 2 & 5.91 & 5.91 & 3.51 & FM \\
\hline IV & 2 & 6.05 & 6.05 & 0.10 & AFM \\
\hline \multicolumn{6}{|c|}{ Substitutional configurations } \\
\hline $\mathbf{a}$ & 2 & 2.43 & 3.83 & 2.95 & FM \\
\hline $\mathbf{b}$ & 2 & 2.79 & 4.19 & 2.92 & FM \\
\hline c & 3 & 2.27 & 3.67 & 1.30 & AFM \\
\hline d & 3 & 2.37 & 3.77 & 1.02 & AFM \\
\hline $\mathbf{e}$ & 4 & 2.09 & 3.49 & 1.53 & AFM \\
\hline $\mathbf{f}$ & 4 & 2.23 & 3.63 & 0.04 & AFM \\
\hline $\mathbf{g}$ & 5 & 2.03 & 3.43 & 1.03 & AFM \\
\hline
\end{tabular}

atom, we investigate all the spin configurations by setting different initial spin configurations on the $\mathrm{Cr}$ atoms. For example, for $n=3$, we consider nonmagnetic, FM ( $\uparrow \uparrow)$ and three AFM or ferrimagnetic states $(\downarrow \uparrow \uparrow, \uparrow \uparrow \downarrow$, and $\downarrow \uparrow \downarrow)$ by setting different initial spin configurations on the $\mathrm{Cr}$ atoms. In this following, AFM also includes ferrimagnetism. The energetically favorable configurations are depicted in Fig. 1 and the net spin moments, the formation energies under $\mathrm{N}$-rich and Al-rich conditions, and the magnetic ground states are listed in Table I.

We first consider doping a single $\mathrm{Cr}$ atom into AlN. Four high-symmetry sites, including two substitutional $\left(\mathrm{Cr}_{\mathrm{Al}}\right.$ and $\mathrm{Cr}_{\mathrm{N}}$ ) and two interstitial sites (tetrahedral $T$ ) and (octahedral $O)\left(\mathrm{Cr}_{i-O}\right.$ and $\left.\mathrm{Cr}_{i-T}\right)$ are considered. The $T$ site corresponds to the center of the line joining the closest nonbonded $\mathrm{Al}$ and $\mathrm{N}$ atoms along the $c$-axis. It has two nearest neighbors (one cation and one anion) and six next-nearest neighbors (three cations and three anions). The $O$ site is located at the center of the hexagonal channel, and has six nearest neighbors (three cations and three anions). As expected, and in agreement with experiments, ${ }^{11,13} \mathrm{Cr}$ atoms are found to prefer substitutional $\mathrm{Al}$ sites, $\mathrm{Cr}_{\mathrm{Al}}$, with an integral magnetic moment of $3 \mu_{\mathrm{B}} / \mathrm{Cr}$. This state is much more energetically favorable than the other three configurations, by more than $6.5 \mathrm{eV} / \mathrm{Cr}$ under $\mathrm{N}$-rich conditions. Under Al-rich growth conditions, the formation energy of $\mathrm{Cr}_{\mathrm{N}}$ is $3.82 \mathrm{eV} / \mathrm{Cr}$ higher than the most favorable $\mathrm{Cr}_{\mathrm{Al}}$ configuration, while the two interstitial sites are even more unfavorable. The calculated value of the magnetic moment is in agreement with previous calculations. ${ }^{15-17,19}$ The other substitutional site, $\mathrm{Cr}_{\mathrm{N}}$, gives an atomic moment of $1.07 \mu_{\mathrm{B}} / \mathrm{Cr}$. For interstitial doping, the $\mathrm{Cr}_{i-O}$ site is lower in energy than the $\mathrm{Cr}_{i-T}$ site by $1.81 \mathrm{eV}$ (32-atom cell) and $1.78 \mathrm{eV}$ (72-atom cell). Both $\mathrm{Cr}_{i-O}$ and $\mathrm{Cr}_{i-T}$ are predicted to be virtually nonmagnetic. The absence 
of magnetism of single $\mathrm{Cr}$ at the interstitial sites may be attributed to the compressed interatomic electron motion ${ }^{28}$ in the limited interstitial region. It was found for $\mathrm{Cr}$ :GaN systems that the formation energies for the substitutionalinterstitial complexes are much higher than the same concentration purely substitutional configurations. ${ }^{20,21}$ Considering the even smaller lattice constants of AlN compared to GaN, the interstitial configurations are expected to play an insignificant role in Cr:AlN. Indeed, the calculated formation energies of these sites for single-Cr doped AlN are all higher than the corresponding values for GaN. Hence, in the following we focus on configurations involving purely substitutional $\mathrm{Cr}_{\mathrm{Al}}$.

For "pair" $\mathrm{Cr}_{\mathrm{Al}}$ doping, the most favorable configuration is that where the two $\mathrm{Cr}$ atoms substitute the in-plane $\mathrm{Al}$ atoms with a distance of $3.14 \AA$ [see Fig. 1(a)]. The next most favorable structure is where the two $\mathrm{Cr}$ atoms substitute nearest-neighbor out-of-plane Al sites [see Fig. 1(b)]. For these two structures, FM coupling is predicted with 32-, 72-, and 96-atom cells, with a moment of $2.92 \mu_{\mathrm{B}} / \mathrm{Cr}$, which is in agreement with recent results. ${ }^{17}$

Significantly, and similar to $\mathrm{Cr}: \mathrm{GaN}$, for the two most favorable configurations of $3-\mathrm{Cr}_{\mathrm{Al}}$-doping systems shown in Figs. 1(c) and 1(d), while using a 72-atom cell predicts a FM ground state with moments of $2.93 \mu_{\mathrm{B}} / \mathrm{Cr}$ and $2.89 \mu_{\mathrm{B}} / \mathrm{Cr}$, larger supercell calculations predict that a state involving AFM coupling is more favorable by 145 and $25 \mathrm{meV}$ per cell (96-atom cell), and 157 and $33 \mathrm{meV}$ per cell (108-atom cell), respectively, resulting in a much lower magnetic moment of $1.30 \mu_{\mathrm{B}} / \mathrm{Cr}$ and $1.02 \mu_{\mathrm{B}} / \mathrm{Cr}$. The magnetic coupling dependence on the size of the supercells demonstrates that for doping $3 \mathrm{Cr}$ atoms in a 72-atom cell, there are still considerable interactions between the periodic neighboring supercells. Note that the smaller supercells tend to favor FM, as found for pair-Cr in AlN (Ref. 17) and poly-Cr in GaN. ${ }^{20,21}$

For higher concentrations of $\mathrm{Cr}_{\mathrm{Al}}$, clustering is still predicted with the AFM state favored. Moreover, the energy difference between the FM and the AFM states increases significantly with the cluster size and reaches $0.61 \mathrm{eV}$ per 96-atom cell, $0.66 \mathrm{eV}$ per 108 -atom cell for the $4-\mathrm{Cr}_{\mathrm{Al}}$ pyramid structure [Fig. 1(e)], resulting in a moment of $1.53 \mu_{\mathrm{B}} / \mathrm{Cr}$. For the in-plane 4-Cr $\mathrm{Al}_{\mathrm{Al}}$ rhombus structure [Fig. $1(\mathrm{f})]$ with a net spin of $0.06 \mu_{\mathrm{B}} / \mathrm{Cr}$, both the 96- and 128atom cells show that the AFM state is lower in energy than the FM state by 62 and $127 \mathrm{meV}$ per cell, respectively. For the $5-\mathrm{Cr}_{\mathrm{Al}}$ bipyramid structure [Fig. $\left.1(\mathrm{~g})\right]$ with a net spin of $1.03 \mu_{\mathrm{B}} / \mathrm{Cr}$, both the 108- and 256-atom cells show that the AFM state is more stable by 0.58 and $0.61 \mathrm{eV}$ per cell. These results clearly demonstrate that as the size of the $\mathrm{Cr}$ clusters increase, AFM coupling becomes more and more favorable. Moreover, from Table I, it is clear that the formation energy per $\mathrm{Cr}$ decreases as the cluster grows, and indicates the energetic preference for the clusters to grow larger. Calculations confirm the formation energy decreases by $0.127 \mathrm{eV} / \mathrm{Cr}$ compared to the one from 96-atom cell.

There are some experimental results showing "Cr-rich clusters" in Cr:AlN. ${ }^{10,14}$ However, the atomic-scale structural information is still unclear. For example, it is difficult to characterize whether they are $\mathrm{Cr}-\mathrm{Cr}$ bonded or $\mathrm{Cr}-\mathrm{N}-\mathrm{Cr}$
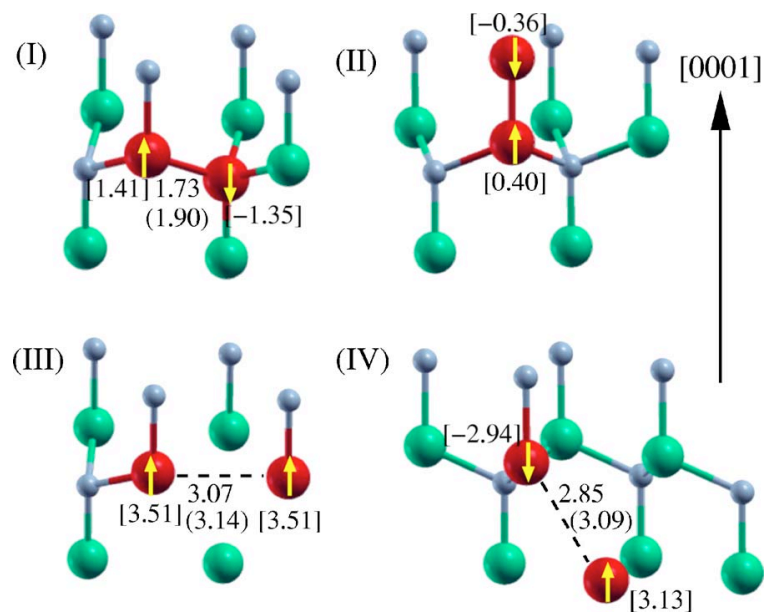

FIG. 2. (Color online) Configurations involving direct $\mathrm{Cr}-\mathrm{Cr}$ interactions. The large dark (red) circles with arrows represent $\mathrm{Cr}$ atoms, the medium light (green) circles represent $\mathrm{Al}$ atoms, and the small light gray circles, $\mathrm{N}$ atoms. Other notations are as in Fig. 1.

bonded within these clusters. In addition, considering that the nearest-neighbor $\mathrm{Cr}-\mathrm{Cr}$ distance in bulk bcc $\mathrm{Cr}$ is $2.49 \AA$, which is between the N-Al (1.90 $)$ ) and the Al-Al (3.14 $)$ distance in AlN, we investigated the possibility of direct $\mathrm{Cr}-\mathrm{Cr}$ bonded clustering, i.e., with no $\mathrm{N}$ atoms in between the two $\mathrm{Cr}$ atoms. Four structures are considered in the 72atom cell, which are depicted in Fig. 2. Both structures I and II contain adjacent $\mathrm{Cr}$ atoms, i.e., $\mathrm{Cr}_{\mathrm{Al}}+\mathrm{Cr}_{\mathrm{N}}$ complexes, where the $\mathrm{Cr}$-atom pairs are parallel and perpendicular to the (0001) plane for structure I and II, respectively. Structures III and IV involve two neighboring $\mathrm{Cr}_{\mathrm{Al}}$ atoms with a $\mathrm{N}$ vacancy in between. The formation energy and magnetic moment for the various magnetic states are listed in Table I. The $\mathrm{Cr}$ atoms exhibit diverse magnetic moments depending on the chemical environment. For the I, II, and IV configurations, AFM states are found favorable over the FM state, while for configuration III, the FM state is energetically more stable than the AFM phase by $85 \mathrm{meV}$ per cell. For the structures I and II shown in Fig. 2, the FM configurations are found to be unstable. Under both $\mathrm{N}$-rich and Al-rich growth conditions, the most favorable configuration is that shown in Fig. 2 (III), where a $\mathrm{N}$ vacancy is present. Overall, the formation energies are, however, much higher than for the two isolated $\mathrm{Cr}_{\mathrm{Al}}$ ions; thus, we conclude that all these $\mathrm{Cr}-\mathrm{Cr}$ bonded configurations are unlikely to play an important role in this system. In addition, our results suggest that the experimentally observed "Cr-rich cluster" is $\mathrm{Cr}-\mathrm{N}-\mathrm{Cr}$ bonded rather than $\mathrm{Cr}-\mathrm{Cr}$ bonded. This is in agreement with recent experimental results indicating that $\mathrm{Cr}-\mathrm{Cr}$ bonding does not exist in $\mathrm{Cr}: \mathrm{GaN}^{29}$

\section{DISCUSSION}

Since the doped magnetic atoms substitute the cation sites and are embedded in the host matrix, such clustering does not violate the wurtzite crystal structure. This may result in it being rather elusive to detect for some experimental techniques. However, clustering does cause a strong local structural distortion, with the $\mathrm{Cr}-\mathrm{Cr}$ distances contracted by up to $14 \%$ in Fig. 1(e) and Fig. 1(g) as compared to the Al-Al 

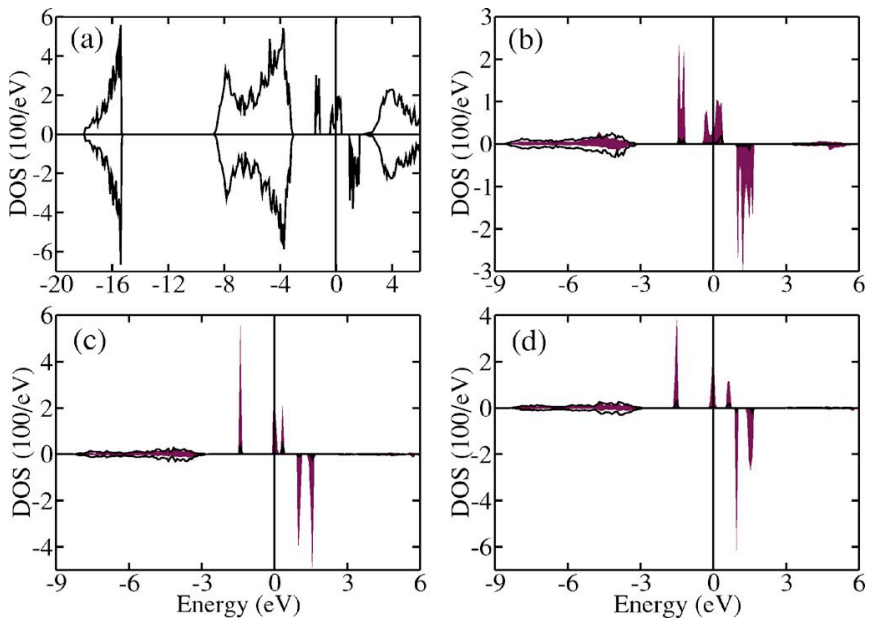

FIG. 3. (Color online) DOS for single $\mathrm{Cr}_{\mathrm{Al}}$ in Cr:AlN: (a) total DOS and (b) the projected DOS as calculated in the 32-atom cell. The projected DOS calculated using a 72-atom cell (c) and in a 96-atom cell (d). In (b), (c), and (d), a smaller energy range is shown as compared to (a). The filled gray areas are for $\mathrm{Cr}-3 d$ states and the solid lines are for the singly $\mathrm{Cr}$-bonded $\mathrm{N}$ atom $2 p$ states.

distance in the host lattice-again indicating a strong attractive interaction between the $\mathrm{Cr}$ atoms. This can be contrasted to the case of a "single" $\mathrm{Cr}_{\mathrm{Al}}$, where the $\mathrm{Cr}-\mathrm{N}$ bond is longer than the ideal $\mathrm{Al}-\mathrm{N}$ bond by $1.7 \%$ due to the larger $\mathrm{Cr}$ atomic radius $(1.40 \AA)$ than that of $\mathrm{Al}(1.25 \AA)$, strong atomic attraction, and magnetic coupling. Interestingly, the lattice constant of the Cr:AlN system is found to systematically decrease with $\mathrm{Cr}$ concentration. ${ }^{11,12}$ This trend may be attributed to the presence of embedded clusters with reduced interatomic distances. Another important feature associated with the clustering is the strong local polarization on the nearby $\mathrm{N}$ atoms; for example, $0.12 \mu_{\mathrm{B}}, 0.15 \mu_{\mathrm{B}}$, and $0.23 \mu_{\mathrm{B}}$ are found in the structures shown in Figs. 1(a), 1(c), and 1(e), respectively, compared to $0.05 \mu_{\mathrm{B}}$ on $\mathrm{N}$ in the isolated $\mathrm{Cr}_{\mathrm{Al}}$ system. The large moments on the $\mathrm{N}$ atoms are due to hybridization between the $\mathrm{N}-2 p$ and $\mathrm{Cr}-3 d$ states.

Now we discuss the electronic structure for which the effect of $\mathrm{Cr}$ concentration plays an important role. Density of states (DOS) plots reveal that isolated $\mathrm{Cr}_{\mathrm{Al}}$ shows halfmetallic character, in which the majority spin state is metallic and the minority spin state is insulating, in all 32-, 72-, and 96-atom cells (i.e., Cr concentrations of $6.25 \%, 2.78 \%$, and $2.08 \%$, respectively); see Fig. 3. However, for the pair-Cr $\mathrm{Cl}_{\mathrm{Al}}$ structure shown in Fig. 1(a), while the 32-atom cell predicts a half-metallic nature, which is in agreement with Ref. 17, both 72- and 96-atom cells predict semiconducting behavior. This dependence of the electronic structure on supercell size can be understood in that the levels in the host band gap are derived from $\mathrm{Cr}-d$ states. Then, for high $\mathrm{Cr}$ concentrations (small cells) there will be significant interactions between the $\mathrm{Cr}$ atoms in neighboring cells, resulting in a broadening of the levels which may result in metallic or semimetallic behavior. In the large supercells, these interactions become very small, giving rise to discrete levels, characteristic of "isolated defects," as can be seen from Fig. 4. For larger clustering configurations [Figs. 1(c) and 1(e)], calculations using the 96-atom cell show that they are both semiconduct-
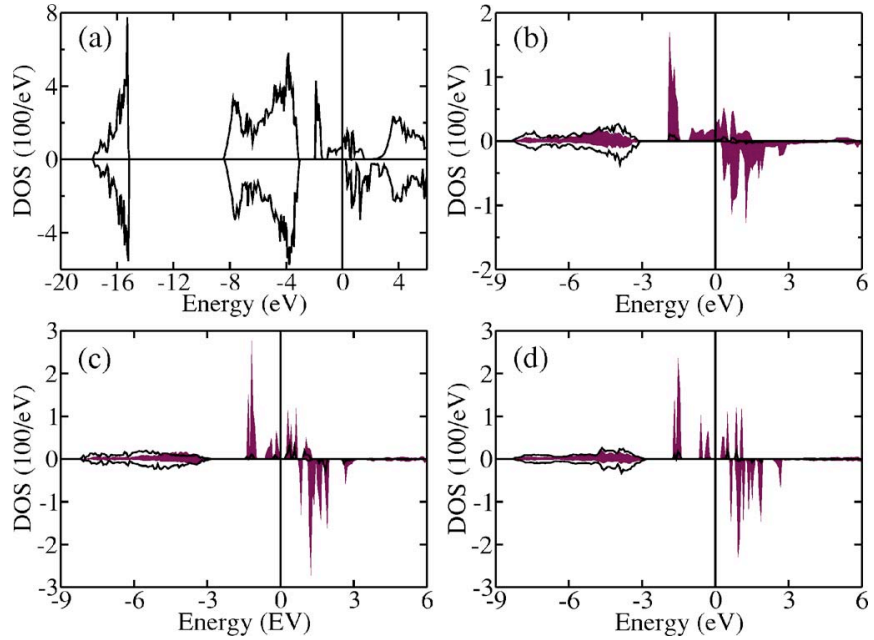

FIG. 4. (Color online) DOS for pair-Cr ${ }_{\mathrm{Al}}$ [cf. Fig. 1(a)] in Cr:AlN: (a) total DOS and (b) the projected DOS as calculated in the 32-atom cell. The projected DOS calculated using a 72-atom cell (c) and in a 96-atom cell (d). In (b), (c), and (d), a smaller energy range is shown as compared to (a). The filled gray areas are for $\mathrm{Cr}-3 d$ states and the solid lines are for the singly Cr-bonded $\mathrm{N}$ atom $2 p$ states.

ing (see Fig. 5). Thus, we propose that the experimentally observed semiconducting or semi-insulating behavior ${ }^{11,14}$ originates from the embedded clustering configurations, rather than isolated $\mathrm{Cr}_{\mathrm{Al}}$.

With increasing $\mathrm{Cr}$ concentration, it has been found experimentally that the mean magnetic moment is strongly suppressed. ${ }^{5,7,11,12}$ This can be understood naturally in that, as the concentration is increased, the $\mathrm{Cr}$ atoms increasingly experience an attractive interaction and form AFM clusters, which results in a lower net magnetization, as intuitively expected by experimentalists. ${ }^{5,10,11}$ More directly, the presence of dopant clustering is strongly supported by recent experiments. For example, while both scanning Auger electron spectroscopy (AES) and secondary ion mass spectrometry (SIMS) profiling of the $\mathrm{Cr}$ atoms did not show any evidence of clustering within the limited resolution of these techniques $(60 \AA$ in the vertical direction for SIMS and $100 \AA$ lateral resolution for the AES), ${ }^{11}$ evidence of $\mathrm{Cr}$ nanosegregation was determined by electron energy loss spectroscopy (EELS) with a much higher resolution of $5 \AA$ probe size. ${ }^{10}$ Moreover, energy-filtered electron micrographs clearly showed that the $\mathrm{Cr}$ atoms form one-dimensional $\mathrm{Cr}$ rich regions, mostly aligned parallel to the growth direction. ${ }^{14}$ It has also been demonstrated that the microstructure and the distribution of the clusters sensitively de-
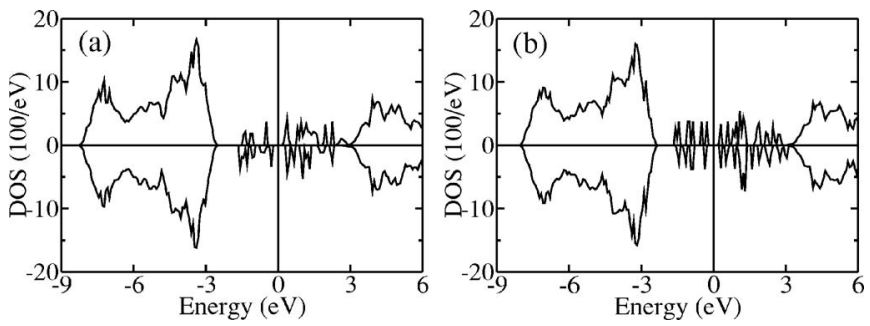

FIG. 5. Total DOS for poly-doping configurations (a) for the structure shown in Figs. 1(c) and (b) for the structure shown in Fig. 1(e), as calculated in the cubic 96-atom cell. 
pend on the substrate temperature: Cr-doped AlN films with a concentration of $4 \%$ grown at $700{ }^{\circ} \mathrm{C}$ show the formation of one-dimensional $\mathrm{Cr}$-rich regions, while growth at $800{ }^{\circ} \mathrm{C}$ results in $\mathrm{Cr}$ atoms forming zero-dimensional $\mathrm{Cr}$-rich spherical clusters, which are randomly distributed within the films. ${ }^{14}$ The preference of the clusters to grow parallel to the growth direction can be understood by considering that the epitaxial samples are grown layer-by-layer along the [0001] direction; consequently, the embedded clusters form much more easily along the $c$-axis than along the $a$-axis due to the attractive interaction. Recently, Fukushima et al. ${ }^{30}$ showed that under layer-by-layer growth conditions, spinodal decomposition leads to quasi-one-dimensional nanostructures for both Cr:ZnTe and Mn:GaN.

The presence of embedded clustering behavior in DMS could be the rule rather than the exception, originating from the attractive interactions among the dopants. Such inhomogeneous distributions will dramatically change the ferromagnetism predicted on the basis of the assumption of a homogeneous or random distribution. We note that recently, embedded clustering behavior was experimentally characterized in precipitate-free (no secondary phases) $\mathrm{Mn}: \mathrm{Ge}$ samples. ${ }^{31,32}$

\section{CONCLUSION}

In summary, we have performed extensive firstprinciples DFT investigations of the spatial distribution and magnetic coupling in Cr-doped AIN. Our systematic studies clearly show that the $\mathrm{Cr}$ ions have a strong tendency to segregate to form $\mathrm{Cr}-\mathrm{N}-\mathrm{Cr}$ bonded embedded clusters. For $\mathrm{Cr}$ clusters larger than 2 atoms, states containing "antiferromagnetic" coupling with net spins in the range $0-1.53 \mu_{\mathrm{B}} / \mathrm{Cr}$ are found to be energetically more favorable. Various cluster configurations are proposed to coexist; the statistical distribution, and consequently the magnetism, will depend sensitively on the growth conditions. The present results serve as a natural explanation to a number of hitherto puzzling experimental observations, notably the low mean magnetic moment, the contracted lattice constants, and the highly insulating behavior. Our proposed embedded clustering is supported by recent high-resolution experiments. We argue that this segregation of TM dopants is a crucial factor in determining the magnetic properties in III-Nitride based DMS and beyond.

\section{ACKNOWLEDGMENTS}

We acknowledge the computing resources provided by the Australian Partnership for Advanced Computing (APAC) National Facility and by the Australian Centre for Advanced Computing and Communications (AC3). We thank the Australian Research Council for financial support. Work at Northwestern University was supported by the NSF (through its MRSEC program).
${ }^{1}$ H. Ohno, Science 281, 951 (1998); S. A. Wolf, D. D. Awschalom, R. A. Buhrman, J. M. Daughton, S. von Molnar, M. L. Roukes, A. Y. Chtchelkanova, and D. M. Treger, ibid. 294, 1488 (2001); I. Žutić, J. Fabian, and S. D. Sarma, Rev. Mod. Phys. 76, 323 (2004).

${ }^{2}$ T. Dietl, H. Ohno, F. Matsukura, J. Cibert, and D. Ferrand, Science 287, 1019 (2000).

${ }^{3}$ V. I. Litvinov and V. K. Dugaev, Phys. Rev. Lett. 86, 5593 (2001).

${ }^{4}$ S. G. Yang, A. B. Pakhomov, S. T. Hung, and C. Y. Wong, Appl. Phys. Lett. 81, 2418 (2002).

${ }^{5}$ D. Kumar, J. Antifakos, M. G. Blamire, and Z. H. Barber, Appl. Phys. Lett. 84, 5004 (2004).

${ }^{6}$ J. Zhang, X. Z. Li, B. Xu, and D. J. Sellmyer, Appl. Phys. Lett. 86, 212504 (2005).

${ }^{7}$ J. Zhang, S. H. Liou, and D. J. Sellmyer, J. Phys.: Condens. Matter 17, 3137 (2005).

${ }^{8}$ R. Frazier, J. Stapleton, G. Thaler, C. R. Abernathy, S. J. Pearton, R. Rairigh, J. Kelly, A. F. Hebard, M. L. Nakarmi, K. B. Nam, J. Y. Lin, H. X. Jiang, J. M. Zavada, and R. G. Wilson, J. Appl. Phys. 94, 1592 (2003).

${ }^{9}$ A. F. Hebard, R. P. Rairigh, J. G. Kelly, S. J. Pearton, C. R. Abernathy, S. N. G. Chu, and R. G. Wilson, J. Phys. D 37, 511 (2004).

${ }^{10}$ S. Y. Wu, H. X. Liu, L. Gu, R. K. Singh, L. Budd, M. van Schilfgaarde, M. R. MacCartney, D. J. Smith, and N. Newman, Appl. Phys. Lett. 82, 3047 (2003).

${ }^{11}$ R. M. Frazier, G. T. Thaler, J. Y. Leifer, J. K. Hite, B. P. Gila, C. R. Abernathy, and S. J. Pearton, Appl. Phys. Lett. 86, 052101 (2005).

${ }^{12}$ A. Y. Polyakov, N. B. Smirnov, A. V. Govorkov, R. M. Frazier, J. Y. Liefer, G. T. Thaler, C. R. Abernathy, S. J. Peartona, and J. M. Zavada, Appl. Phys. Lett. 85, 4067 (2004).

${ }^{13}$ H. X. Liu, S. Y. Wu, R. K. Singh, L. Gu, D. J. Smith, N. Newman, N. R. Dilley, L. Montes, and M. B. Simmonds, Appl. Phys. Lett. 85, 4076 (2004).

${ }^{14}$ L. Gu, S. Y. Wu, H. X. Liu, R. K. Singh, N. Newman, and D. J. Smith, J. Magn. Magn. Mater. 290-291, 1395 (2005).

${ }^{15}$ K. Sato and H. Katayama-Yoshida, Jpn. J. Appl. Phys., Part 2 40, L485 (2001).

${ }^{16}$ J. E. Medvedeva, A. J. Freeman, X. Y. Cui, C. Stampfl, and N. Newman, Phys. Rev. Lett. 94, 146602 (2005).

${ }^{17}$ Q. Wang, A. K. Kandalam, Q. Sun, and P. Jena, Phys. Rev. B 73, 115411 (2006).

${ }^{18}$ P. Mahadevan, J. M. Osorio-Guillen, and A. Zunger, Appl. Phys. Lett. 86, 172504 (2005).

${ }^{19}$ M. van Schilfgaarde and O. N. Mryasov, Phys. Rev. B 63, 233205 (2001).

${ }^{20}$ X. Y. Cui, J. E. Medvedeva, B. Delley, A. J. Freeman, N. Newman, and C. Stampfl, Phys. Rev. Lett. 95, 256404 (2005).

${ }^{21}$ X. Y. Cui, J. E. Medvedeva, B. Delley, A. J. Freeman, and C. Stampfl, Phys. Rev. B 75, 155205 (2007).

${ }^{22}$ J. P. Perdew, K. Burke, and M. Ernzerhof, Phys. Rev. Lett. 77, 3865 (1996).

${ }^{23}$ B. Delley, J. Chem. Phys. 113, 7756 (2000); 92, 508 (1990).

${ }^{24}$ B. Delley, Phys. Rev. B 66, 155125 (2002).

${ }^{25}$ C. Stampfl and C. G. Van de Walle, Phys. Rev. B 59, 5521 (1999), and references therein.

${ }^{26}$ J. M. Simmons, Metall. Mater. Trans. A 26, 2579 (1995).

${ }^{27}$ Handbook of Chemistry and Physics, edited by R. C. Weast, M. J. Astle, and W. H. Beyer (Chemical Rubber Co., Boca Raton, FL, 1988), Vol. 62.

${ }^{28}$ V. L. Moruzzi, Phys. Rev. Lett. 57, 2211 (1986).

${ }^{29}$ T. Takeuchi, Y. Harada, T. Tokushima, M. Taguchi, Y. Takata, A. Chainani, J. J. Kim, H. Makino, T. Yao, T. Yamamoto, T. Tsukamoto, S. Shin, and K. Kobayashi, Phys. Rev. B 70, 245323 (2004).

${ }^{30}$ T. Fukushima, K. Sato, H. Katayama-Yoshida, and P. H. Dederichs, Jpn. J. Appl. Phys., Part 2 45, L416 (2006).

${ }^{31}$ A. P. Li, J. F. Wendelken, J. Shen, L. C. Feldman, J. R. Thompson, and H. H. Weitering, Phys. Rev. B 72, 195205 (2005).

${ }^{32}$ D. Bougeard, S. Ahlers, A. Trampert, N. Sircar, and G. Abstreiter, Phys. Rev. Lett. 97, 237202 (2006). 